\title{
The effect of B chromosomes on outcrossing rate in a population of rye, Secale cereale L.
}

\author{
M. Cruz-Pardilla, \\ F. J. Vences, \\ P. Garcia and \\ M. Pérez de la Vega.
}

Departamento de Genética, Facultad de Biología, Universidad de León, E-24071 León, Spain.

The effect of B chromosomes (Bs) on the mating system of a population of Secale cereale $\mathbf{L}$. was studied. The population was formed with one half of the plants bearing Bs and the other half without Bs. Outcrossing rates were estimated by both a single-locus method and a multilocus method using allozyme loci as genetic markers. The rate of selfing in this population was high, close to 35 per cent, but there are differences between plants with and without Bs. While the subpopulation of plants with Bs showed a selfing rate close to 20 per cent, the subpopulation of plants without Bs reached a value of close to 45 per cent. Differences in allele frequencies at some loci between the two subpopulations of plants have been also observed. Our data reinforce the previously known effects of Bs in influencing mating system and the genetic structure of rye populations.

\section{INTRODUCTION}

B chromosomes are characterized by their dispensable nature to normal growth and development of individuals, by their non-Mendelian mode of transmission and by their deleterious effects when present in large numbers. Bs are transmitted following species-specific rules and the mechanisms of transmission tend to lead to their accumulation in individuals. This accumulation is compensated by the generally lower fitness of individuals carrying a high number of Bs (Jones and Rees, 1982).

The mating system has important effects on the genetic structure of plant populations, since it is a major factor determining the amount, distribution and maintenance of genetic variation in populations (Allard et al., 1975).

Rye (Secale cereale L.) is a plant species which naturally carries $\mathrm{Bs}$ in some of its populations (Jones and Rees, 1982). Its mating system is predominantly outcrossing but, in spite of the existence of a multiallelic two-locus incompatibility system (Lundqvist, 1956; Trang et al., 1982), selfing occurs, ranging from 0 to 30 per cent (Pérez de la Vega and Allard, 1984; Vaquero, 1987; Vaquero et al., 1989), although higher values have been reported in synthetic populations with selffertility genes (Wricke, 1979).
It has been pointed out that pollen from rye plants with Bs fecundates more frequently than pollen from rye plants without Bs (Puertas and Carmona, 1976). Thus the presence of Bs could play an important role on the mating system of populations.

The principal aims of the present work were to ascertain the effect of Bs on the selfing rate of rye plants, and to determine the effect of these chromosomes on the genetic structure of rye populations. To do so we have studied subpopulations with and without Bs of a population of JNK, a cultivar of rye naturally bearing Bs. Previous results (García et al., 1982) indicated that selfpollination of JNK plants was possible, so this cultivar would probably have a measurable rate of selfing in natural pollination conditions.

\section{MATERIALS AND METHODS}

The material used was a population of the Japanese cultivar JNK of diploid rye (Secale cereale L., $2 n=14+$ Bs). A large sample of seeds was kindly supplied by Dr Kishikawa, and the population has been maintained and replanted several times in Spain. Approximately 1000 seeds of rye were simultaneously germinated on wet paper filter 
under controlled conditions, chromosome numbers were determined by root tip screening after ice-cool water treatment, fixation in acetic acid:alcohol $1: 3$ and staining by the Feulgen method. Table 1 shows the percentages of plants with the different chromosome numbers obtained. Leaves of a random sample (bulk sample) of 375 individuals from the chromosomally controlled seedlings were excised after 15 days of germination, crushed and the crude extracts electrophoresed in starch gels; after electrophoresis the genotypes of isozymatic loci (see below) were scored. S. vavilovii homozygous plants were used as standard in gels.

After chromosome counting 500 seedlings were randomly chosen, 250 of them carrying Bs and 250 without Bs (table 1). Hereafter we shall designate these two sets as subpopulation with or without Bs, respectively. These 500 seedlings were transplanted to a rectangular field ( 27 rows $\times 19$ columns) and planted randomly at $30 \mathrm{~cm}$ of separation from each other. 497 plants survived to maturity. All these plants were allowed to interpollinate freely. Furthermore, about 150 additional seedlings, with 0,2 or $4 \mathrm{~B}$ chromosomes, were transplanted to big pots and at flowering time the plants were selfed by bagging the spikes of each plant.

Isozymatic loci were used to estimate the genetic structure and outcrossing rates of rye population and subpopulations. The isozymatic systems scored were PGI, PGM, 6PGD, GOT, MDH, and ACP. Electrophoretic procedures and genetic nomenclature have been described elsewhere (Pérez de la Vega and Allard, 1984; Vaquero, 1987; Rebordinos and Pérez de la Vega, 1988).

After the free pollination of the 497 surviving plants, 200 plants were randomly chosen, 100 with Bs and 100 without Bs, a single spike of each plant

Table 1 Percentages of plants with different B chromosome numbers

\begin{tabular}{lcc}
$\begin{array}{l}\text { Number of } \\
\text { B chromosomes }\end{array}$ & $\begin{array}{l}\text { Of the total of } \\
\text { plants scored }\end{array}$ & $\begin{array}{l}\text { Of the plants } \\
\text { transplanted } \\
\text { to fields }\end{array}$ \\
\hline 0 & 36.90 & $50 \cdot 30$ \\
1 & $5 \cdot 96$ & $5 \cdot 23$ \\
2 & 31.65 & 23.94 \\
3 & $6 \cdot 37$ & 4.63 \\
4 & 17.68 & $15 \cdot 09$ \\
5 & 0.21 & $0 \cdot 00$ \\
6 & 1.23 & 0.80 \\
Total No. & & \\
of plants & 973 & 497
\end{tabular}

was excised and 10 seedlings per spike were analyzed electrophoretically. The genetic data of these progeny arrays were used to obtain single locus (Clegg et al., 1978) and multilocus (Shaw et al., 1981) estimates of the outcrossing rate $(t)$ in the subpopulations with and without $\mathrm{Bs}$, and in the whole population. Isozyme loci genotypes of each maternal plants were inferred from the genotypes of progeny arrays by the method of Clegg et al. (1978). The weighted mean of single locus estimates was calculated according to Kahler et al. (1984).

\section{RESULTS}

The B chromosome number of JNK individual ranged from 0 to 6 in our population (table 1). In addition some individuals with iso-Bs were observed in our screening of the population, but they were not considered for the populational study. The frequency of individuals with Bs was $63 \cdot 10$ per cent, and among them the individuals with even numbers of Bs outnumbered four to one the individuals with odd numbers (table 1).

Table 2 shows the genotype and allelle frequencies of the variable loci in the bulk sample of seedlings from the maternal generation. In addition to these loci three other loci showed no variability in this population: Got-2, Mdh-1 and 6-Pgd1. Zone GOT1 is highly variable but since it is under the control of a duplicate gene (Rebordinos and Pérez de la Vega, 1988) it is not always possible to know the genotype of plants from the observation of the electrophoretic patterns; so this zone was not included in our study. All variable loci showed two codominant alleles, except for Pgi-2 which had three, but two of them were scored as a single composite allele to avoid mistakes in scoring genotypes due to the close migration of isozymes in gels. Among these six variable loci only four are polymorphic while for the other two ( $\mathrm{Pgm}$, $M d h-2)$ the frequency of the most frequent allele is over 0.99 . Three of the loci showed a significant deviation of their genotypic frequencies from the expectations under Hardy-Weinberg randommating equilibrium; these deviations were observed in the whole sample and in one of the subpopulations with or without Bs (table 2), and were always associated with a number of heterozygotes lower than expected. Significant differences in allelic frequencies of $G o t-3$ and $A c p-2$ loci were observed between the subpopulation with Bs and the subpopulation without Bs (table 2). The observed heterozygosity mean was $0 \cdot 226 \pm 0 \cdot 073$ 
Table 2 Observed frequencies in the bulk sample of JNK seedlings

\begin{tabular}{|c|c|c|c|c|c|}
\hline \multirow[b]{2}{*}{ Locus } & & \multicolumn{3}{|l|}{ Genotypic } & \multirow[b]{2}{*}{ Allelic* } \\
\hline & & 11 & 12 & 22 & \\
\hline \multirow[t]{3}{*}{$P g m$} & $\mathrm{~T}^{\dagger}$ & 0.989 & 0.011 & 0.000 & $0.995(0.003)$ \\
\hline & 0 & 0.988 & 0.012 & 0.000 & $0.994(0.004)$ \\
\hline & B & 0.991 & $0 \cdot 009$ & $0 \cdot 000$ & $0.995(0.003)$ \\
\hline \multirow[t]{3}{*}{$P g i-2$} & $\mathrm{~T}$ & $0.565^{* * *} \S$ & 0.328 & $0 \cdot 107$ & $0.729(0.016)$ \\
\hline & 0 & $0.593^{* * *}$ & $0 \cdot 265$ & $0 \cdot 142$ & $0.725(0.025)$ \\
\hline & B & $0 \cdot 545$ & $0 \cdot 375$ & $0 \cdot 080$ & $0.732(0.021)$ \\
\hline \multirow[t]{3}{*}{ 6-Pgd-2 } & $\mathrm{T}$ & $0 \cdot 616^{* *}$ & $0 \cdot 307$ & 0.077 & $0.769(0.015)$ \\
\hline & 0 & 0.654 & $0 \cdot 290$ & $0 \cdot 056$ & $0.799(0.022)$ \\
\hline & B & $0.587^{*}$ & $0 \cdot 319$ & $0 \cdot 094$ & $0.746(0.021)$ \\
\hline \multirow[t]{3}{*}{ Got-3 } & $\mathrm{T}$ & 0.416 & 0.437 & $0 \cdot 147$ & $0.635(0.018)$ \\
\hline & 0 & $0 \cdot 296$ & 0.506 & $0 \cdot 198$ & $0.549(0.028) \|$ \\
\hline & B & $0 \cdot 507$ & $0 \cdot 385$ & $0 \cdot 108$ & $0.700(0.022)$ \\
\hline \multirow[t]{3}{*}{ Acp-2 } & $\mathrm{T}$ & $0.659^{* * *}$ & 0.269 & 0.072 & $0.793(0.105)$ \\
\hline & 0 & $0 \cdot 562^{*}$ & $0 \cdot 321$ & $0 \cdot 177$ & $0.722(0.025) \|$ \\
\hline & B & $0 \cdot 732$ & $0 \cdot 230$ & 0.038 & $0 \cdot 847(0.017)$ \\
\hline \multirow[t]{3}{*}{$M d h-2$} & $\mathrm{~T}$ & 0.992 & 0.003 & 0.005 & $0.993(0.003)$ \\
\hline & 0 & 0.988 & 0.000 & 0.012 & $0.988(0.006)$ \\
\hline & $\mathrm{B}$ & 0.995 & 0.005 & 0.000 & $0.998(0.002)$ \\
\hline
\end{tabular}

* Frequency of allele number 1 (standard deviation, $\sigma$ ).

$\dagger \mathrm{T}=$ Total scored (375 seedlings); $0=$ Subpopulation without B chromosomes (162 seedlings); $\mathrm{B}=$ subpopulation with $\mathrm{B}$ chromosomes (213 seedlings).

$\ddagger$ Allele here designated as 1 is according to Pérez de la Vega and Allard (1984) a composite allele of the alleles 1 and 2 , and allele 2 corresponds to the allele they designated 3 .

$\S$ Chi-square significance in relation to Hardy-Weinberg expectations; $* P<0.05, * * P<$ $0 \cdot 01, * * * P<0 \cdot 001$.

$\|$ Significant difference between the allelic frequencies of the subpopulations with and without B chromosomes.

for the six variable loci, and $0 \cdot 151 \pm 0 \cdot 060$ for the nine loci scored.

A population of 500 seedlings (table 1) was transplanted to a field and, after free pollination, a random sample of 200 mature spikes was collected, 100 from plants without Bs and 100 from plants with Bs. The genotypes of these plants were inferred from the genotypes of 10 seedlings of each of the 200 progenies according with the method of Clegg et al. (1978). Non-significant differences in genotypic and allelic frequencies were observed between this sample of 200 adult plants and the bulk sample previously analyzed at seedling stage. The same was true when comparing the subpopulations with and without Bs at seedling and adult stages. The differences in allelic frequencies of Got-3 and Acp-2 between both subpopulations were also observed at the adult stage.

The genotypic and allelic frequencies of the 2000 descendant seedlings, together and separate as progenies from mother plants with or without Bs, are shown in table 3. Five of the loci showed a significant deviation of genotypic frequencies from random-mating expectations in the whole population and in the subpopulations of plants without.Bs, furthermore, two of these loci also showed a significant deviation in the subpopulation from plants with Bs (table 3 ). In all of the loci these deviations were always due to an almost symmetrical excess of both homozygotes over the expected values under random mating. In this generation the differences in allelic frequencies between the subpopulations from plants with and without Bs were also significant for $6-P g d-2$ and $M d h-2$, in addition to that observed for Got-3 and $A c p-2$ in the previous generation (table 3 ). The mean heterozygosities, estimated from the observed data, for the six variable loci or for the nine loci were as follows: $0 \cdot 160 \pm 0 \cdot 060$ and $0 \cdot 142 \pm$ $0 \cdot 056$ for the total of seedlings, $0 \cdot 154 \pm 0 \cdot 059$ and $0 \cdot 137 \pm 0 \cdot 055$ for the seedlings from mother plants without Bs, and $0 \cdot 166 \pm 0.062$ and $0 \cdot 147 \pm 0.058$ for the seedlings from mother plants with Bs.

Outcrossing rates $(t)$ were estimated locus to locus by means of the single locus method (Clegg et al., 1978) and then a weighted mean was 
Table 3 Observed frequencies in the seedling progenies from plants with and without $\mathrm{B}$ chromosomes

\begin{tabular}{|c|c|c|c|c|c|}
\hline \multirow[b]{2}{*}{ Locus } & & \multicolumn{3}{|l|}{ Genotypic } & \multirow[b]{2}{*}{ Allelic* } \\
\hline & & 11 & 12 & 22 & \\
\hline \multirow[t]{3}{*}{$P g m$} & $T^{\dagger} \div$ & 0.982 & 0.017 & 0.001 & $0.990(0.001)$ \\
\hline & 0 & 0.983 & 0.017 & 0.000 & $0.992(0.002)$ \\
\hline & B & 0.980 & 0.018 & 0.002 & $0.989(0.002)$ \\
\hline \multirow[t]{3}{*}{ Pgi-2‡ } & $\mathrm{T}$ & $0.609^{* * *} \S$ & $0 \cdot 313$ & $0 \cdot 078$ & $0.765(0.007)$ \\
\hline & 0 & $0.615^{* * *}$ & $0 \cdot 286$ & 0.099 & $0.758(0.010)$ \\
\hline & $B$ & 0.603 & 0.339 & 0.058 & $0.773(0.009)$ \\
\hline \multirow[t]{3}{*}{ 6-Pgd-2 } & $\mathrm{T}$ & $0.652^{* * *}$ & 0.274 & 0.074 & $0.789(0.006)$ \\
\hline & 0 & $0.685^{* * *}$ & $0 \cdot 240$ & 0.075 & $0.805(0.009) \|$ \\
\hline & B & $0.619^{* * *}$ & $0 \cdot 308$ & 0.073 & $0.773(0.009)$ \\
\hline \multirow[t]{3}{*}{ Got-3 } & $\mathrm{T}$ & $0.410^{* * * *}$ & 0.413 & 0.177 & $0.616(0.008)$ \\
\hline & 0 & $0.348^{* * *}$ & $0 \cdot 416$ & 0.236 & $0.556(0.011) \|$ \\
\hline & B & $0 \cdot 471$ & 0.411 & 0.118 & $0.677(0.010)$ \\
\hline \multirow[t]{3}{*}{ Acp-2 } & $\mathrm{T}$ & $0.677^{* * *}$ & 0.253 & 0.070 & $0.803(0.006)$ \\
\hline & 0 & $0.629^{* * *}$ & $0 \cdot 265$ & $0 \cdot 106$ & $0.762(0.009)$ \\
\hline & B & $0.724 *$ & $0 \cdot 242$ & 0.034 & $0.845(0.008)$ \\
\hline \multirow[t]{3}{*}{$M d h-2$} & $\mathrm{~T}$ & $0.986^{* * *}$ & 0.006 & 0.008 & $0.989(0.002)$ \\
\hline & 0 & $0.979^{* * *}$ & 0.005 & 0.016 & $0.982(0.003) \|$ \\
\hline & $\mathrm{B}$ & 0.993 & 0.006 & 0.001 & $0.996(0.001)$ \\
\hline
\end{tabular}

* Frequency of allele number 1 (standard deviation, $\sigma$ ).

$\dagger \mathrm{T}=$ Total scored (2000 seedlings); $0=$ Subpopulation without $\mathrm{B}$ chromosomes (1000 seedlings); $\mathrm{B}=$ subpopulation with $\mathrm{B}$ chromosomes (1000 seedlings).

$¥$ Allele here designated as 1 is according to Pérez de la Vega and Allard (1984) a composite allele of the alleles 1 and 2, and allele 2 corresponds to the allele they designated 3 .

$\S$ Chi-square significance in relation to Hardy-Weinberg expectations; $* P<0.05$, *** $P<$ $0 \cdot 001$.

$\|$ Significant difference between the allelic frequencies of the subpopulations with and without B chromosomes.

calculated from the single locus estimates according to Kahler et al. (1984); and also estimated by means of the multilocus method of Shaw et al. (1981), which yield a single value from the simultaneous analysis of all the loci. The same samples of individuals were used in both methods. Three pairs of estimations were carried out (table 4). For the subpopulation with Bs the 100 progeny arrays of 10 seedlings per mother plant with Bs were used; the estimates for the subpopulation without Bs were obtained in a separate analysis from the 100 progeny arrays of mother plant lacking $\mathrm{Bs}$; and the whole population estimates were obtained from the 200 progenies jointly analyzed. The values obtained by means of the multilocus method and the weighted mean of the single locus estimates were similar for each group of plants (table 4), but significantly different between groups. Thus, according to the multilocus method which showed slightly higher estimates of outcrossing, the selfing rate $(s=1-t)$ for the JNK rye should be close to 35 per cent, while the plants with and without Bs should have selfing rates close to $20 \%$ and $45 \%$, respectively.

Fertility, estimated as a percentage of seed set per plant, was scored in a random sample of 50 plants taken from all plants in the population, and in three samples of 50 plants each with 0,2 or $4 \mathrm{~B}$ chromosomes. The percentages of seed sets were:

Table 4 Weighted mean of single-locus estimations and multilocus estimations of outcrossing rates in plants with and without $\mathrm{B}$ chromosomes

\begin{tabular}{llll}
\hline & $\begin{array}{l}\text { Single-locus } \\
\text { weighted } \\
\text { mean* }\end{array}$ & Multilocus & $\begin{array}{l}\text { Number of } \\
\text { mother } \\
\text { plants }\end{array}$ \\
\hline $\begin{array}{c}\text { Without B } \\
\text { chromosomes }\end{array}$ & $0.546(0.045)$ & $0.554(0.023)$ & 100 \\
$\begin{array}{c}\text { With B } \\
\text { chromosomes }\end{array}$ & $0.726(0.065)$ & $0.796(0.027)$ & 100 \\
Both groups & $0.612(0.034)$ & $0.648(0.017)$ & 200 \\
\hline
\end{tabular}

* Standard deviations in parentheses.

$\uparrow 10$ progeny seedlings per each mother plant were scored. 

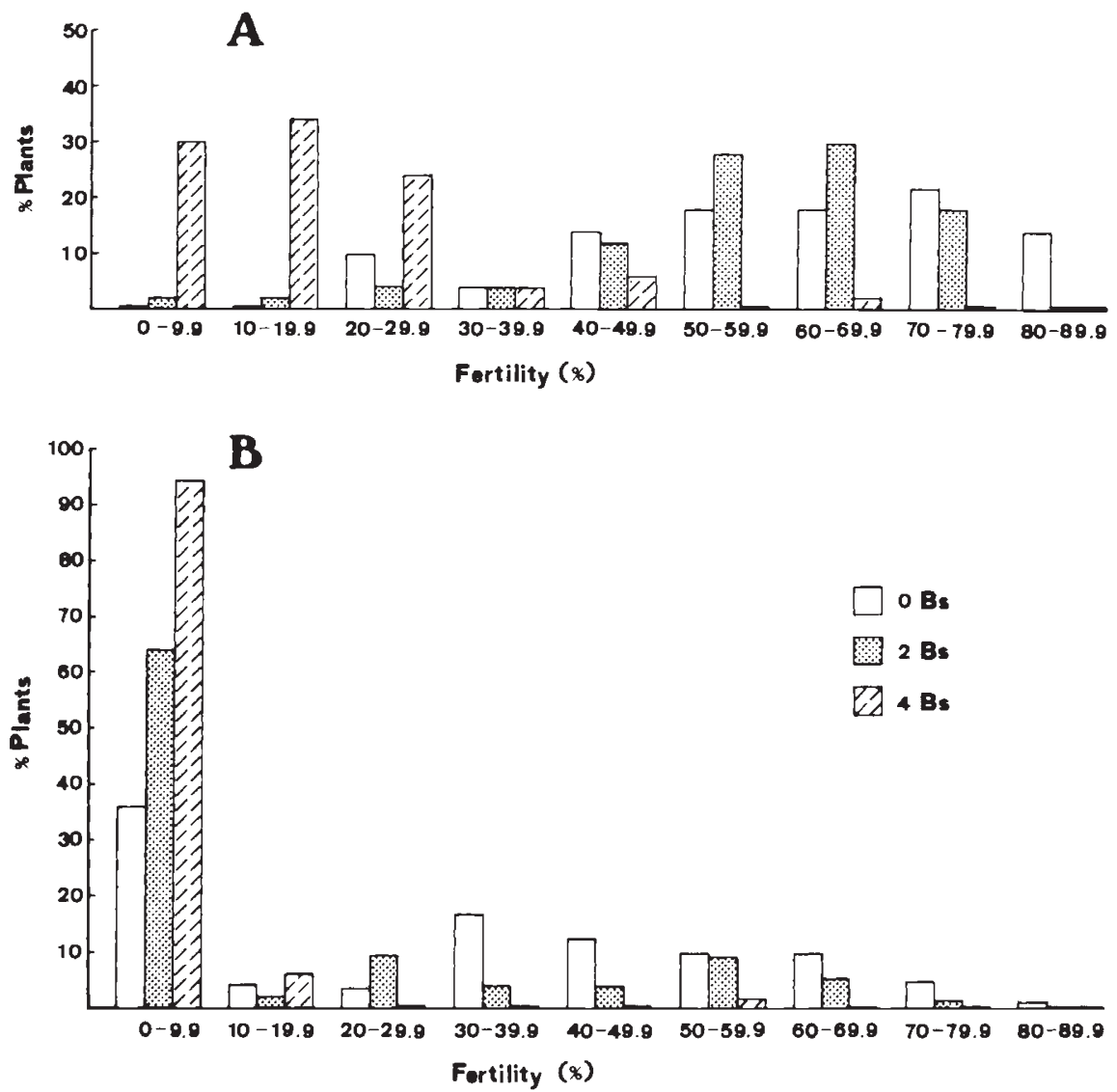

Figure 1 Distribution of fertility (percentage of flowers with seed) among JNK plants with 0,2 or 4 B chromosomes. (A), openpollinated population; (B), self-pollinated plants.

56.24 per cent (ranging among plants from 1.64 to 89.66 per cent) for the population sample; 61.93 per cent $(20 \cdot 75$ to $89 \cdot 64$ per cent $)$ for plants without Bs; 57.98 per cent $(4.03$ to 77.65 per cent) for plants with $2 \mathrm{~B}$ chromosomes; and 18.52 per cent ( 1.53 to $62 \cdot 15$ per cent) for plants with $4 \mathrm{~B}$ chromosomes (fig. 1(A)). In addition to these plants from the free-pollinated field population, a set of plants grown in pots was selfed bagging all the spikes of each plant together. Fertilities of these selfed plants were 30.79 per cent $(0.00$ to 75.00 per cent $)$, 17.53 per cent $(0.00$ to 76.51 per cent $)$, and 0.79 per cent $(0.00$ to 17.31 per cent $)$, respectively for plants with 0,2 or $4 \mathrm{~B}$ chromosomes (fig. 1(B)).

\section{DISCUSSION}

JNK rye is similar to other rye cultivars so far analyzed on the basis of allozyme loci since the alleles present in JNK are present in all other studied rye cultivars, and their frequencies are similar in relation to other cultivars (Pérez de la Vega and Allard, 1984; Ramírez et al., 1985; Vaquero et al. 1989).

The more noticeable result in relation to JNK allelic frequencies is the significant differences observed between the subpopulations with and without Bs for the loci Got-3 and Acp-2 in the bulk sample, and Got-3, Acp-2, 6-Pgd-2 and Mdh2 in the progenies from these two subpopulations after free pollination in the field. We have not found an explanation for this result on the basis of our experiments. The population was kept up by planting random samples of seed of unknown isozyme genotype and chromosomal constitution, and isolated from other rye populations; the year when this experiment was carried out, JNK was the only rye population in our experimental field. Extracts from seedlings with and without Bs were electrophoresed in the same gels $(20$ samples per gel). A possible explanation could be a differential 
selection of allozyme genotypes depending upon the presence or absence of Bs, but this hypothesis has to be properly tested.

In general, less heterozygotes were found at all polymorphic loci than expected in random mating populations, and the observed average heterozygosity was lower than the expected one on the assumption of panmixia. Deficiences for heterozygotes have also been described in other rye cultivars and attributed to selfing (Pérez de la Vega and Allard, 1984; Vaquero et al., 1989). The rates of selfing detected in JNK could be the main cause of the defect of heterozygotes detected in this rye population.

Both methods of outcrossing estimates yield similar results in all cases. The selfing rate of the subpopulation of plants without Bs was 45 per cent, while the selfing rate of the plants with Bs gave an estimate of 20 per cent, obtained by the multilocus method. When the outcrossing rate was calculated with both subsets of plants together the selfing rate was near to 35 per cent.

Although rye is a species with a multiallelic two-locus self-incompatibility system (Lundqvist, 1956: Trang et al., 1982) the incompatibility barrier can be overcome by the presence of self-fertility genes and/or by pseudocompatibility due to the misfunctioning of the incompatibility system caused by environmental factors (Wricke, 1974, 1979; Schmidt-Stohn et al., 1986). Selfing rates up to 40 per cent have been indirectly estimated in synthetic self-compatible rye populations, and rates of up to 25 per cent have been reported because of pseudocompatibility (Wricke 1974, 1979). Estimations in natural populations planted at normal density ranged from 0 to 30 per cent (Vaquero et al., 1989). The main cause of selfing in JNK seems to be due to the presence of selffertility genes since 64.4 per cent of the bagged plants without Bs showed a fertility higher than 10 per cent (fig. $1(\mathrm{~B})$ ).

Plants without Bs showed a selfing rate twice that of plants with Bs, in spite of the fact that the pollen pool was the same for both kinds of plants. This represents a drastic effect of the Bs on the mating system. The effect of Bs could be due to one or both of the following hypotheses: (i) Bs act directly on the incompatibility genetic system reinforcing the pollen self-incompatibility reaction, even in plants with self-fertility genes; and/or (ii) act indirectly because the zygotes formed by self-pollination in plants with two or more Bs frequently have a higher number of $\mathrm{Bs}$ and therefore lower viability than zygotes with $0-2$ B chromosomes, thus the proportion of seed set resulting from cross-pollination would be higher in plants with Bs than in plants lacking in them.

The first hypothesis is only indirectly supported by the known effect of Bs on other rye phenotypic characters (Müntzing, 1963; Jones and Rees, 1982), but there is no direct experimental evidence of an effect of Bs on the genetic system controlling incompatibility in rye; although it has been suggested that the presence or absence of Bs in the style and pollen could determine the probability of a pollen grain in being involved in fertilization (Puertas et al., 1986).

The second hypothesis is supported by our results which show that fertility of plants decreases as the number of Bs increases. In the open-pollinated population there is only a slight diminution (6.4 per cent) for plants with Bs, in relation to plants without them, but the reduction in fertility $(70.1$ per cent) is more noticeable among plants with 4B chromosomes. In an open-pollinated population a proportion of zygotes on plants with Bs is formed by the fertilization of pollen without Bs coming from other plants of the population; but in selfed plants, due to the post-meiotic accumulation mechanism of $\mathrm{Bs}$, a great proportion of zygotes has a number of Bs higher than the number of their mother plant. Thus, according to the second hypothesis a higher decrease in fertility is expected in selfed plants with Bs in comparison to both, selfed plants without Bs and open-pollinated plants with Bs. Our results agree with that expected, the diminutions of fertility in selfed plants with 2 and $4 \mathrm{~B}$ chromosomes were respectively of 43.1 and 97.4 per cent in comparison to selfed plants without Bs. Progressive reductions in fertility as the number of Bs increases have been previously reported in open and self-pollinated plants of rye (Jones and Rees, 1982; Puertas et al., 1985).

B chromosomes seem to play an important role in the mating system of rye populations. They appear to promote mating among different plants either directly or indirectly, at least in populations where self-fertilization is possible.

Acknowledgements This work has been supported by grants (PR82-1789 and PB85-0153) from the C.I.C.Y.T. of Spain.

\section{REFERENCES}

AllARI, R. W., KAHLER, A. L. AND ClEGG, M. T. 1975. Isozymes in plant population genetics. In Market, C. L. (ed.) Isozymes IV, Academic Press, New York. 
CLEGG, M. T., KAHLER, A. L. AND ALLARD, R. W. 1978. Estimation of life cycle components of selection in an experimental plant population. Genetics, 89, 765-792.

GARCIA, P., PEREZ DE LA VEGA, M. AND BENITO C. 1982. The inheritance of rye seed peroxidases. Theor. Appl. Genet., $61,341-351$.

JONES, R. N. AND REES, H. 1982 B Chromosomes. Academic Press, London.

KAHLER, A. L., GARDNER, C. O. AND ALlARD, R. W. 1984 Nonrandom mating in experimental populations of maize. Crop Sci., 72, 943-946.

LUNDQVIST, A. 1956. Self-incompatibility in rye I. Genetic control in the diploid. Hereditas, 42, 293-348.

MÜNTZING, A. 1963. Effect of accessory chromosomes in diploid and tetraploid rye. Hereditas, 49, 371-426.

PEREZ DE LA VEGA, M. AND AllaRD, R. W. 1984. Mating system and genetic polymorphism in populations of Secale cereale and S. vavilovii. Can. J. Genet. Cytol., 26, 308-317.

PUERTAS, M. J., BAEZA, F. AND DE LA PEÑA, A. 1986. The transmission of B chromosomes in populations of Secale cereale and Secale vavilovii 1 . Offspring obtained from $\mathrm{OB}$ and 2B plants. Heredity, 57, 389-394.

PUERTAS, M. J. AND CARMONA, R. 1976. Greater ability of pollen tube growth in rye plants with $2 \mathrm{~B}$ chromosomes. Theor. Appl. Genet., 47, 41-43.

PUERTAS, M. J., ROMERA, F. AND DE LA PEÑA, A. 1985. Comparison of $\mathrm{B}$ chromosome effects on Secale cereale and Secale vavilovii. Heredity, 55, 229-234.
RAMIREZ, L. PISABARRO, G. AND PEREZ DE LA VEGA, M. 1985 Isozyme genetic similarity among rye (Secale cereale L.) cultivars. J. Agric. Sci., 105, 495-500.

REBORDINOS, L. AND PEREZ DE LA VEGA, M. 1988. Gene duplication in the structural gene for a glutamate oxaloacetate transaminase (GOT 1) zone in Secale. J. Hered., 79, $78-80$.

SCHMIDT-STOHN, G., WRICKE, G. AND WEBER, W. E. 1986 Estimation of selfing rates in self-fertile rye plants using isozyme marker loci. Z. Pflanzenzüchtg., 96, 181-184.

SHAW, D. V., KAHLER, A. L. AND ALlARD, R. W. 1981. A multilocus estimator of mating system parameters in plant populations. Proc. Natl. Acad. Sci., 78, 1289-1302.

TRANG, Q. S. WRICKE, G. AND WEBER, W. E. 1982. Number of alleles at the incompatibility loci in Secale cereale $\mathrm{L}$. Theor. Appl. Genet., 63, 245-248.

VAQUero, F. 1987. PhD. Thesis. Universidad Complutense de Madrid.

VAQUERO, F., VENCES, F. J., GARCIA, P., RAMIREZ, L. AND PEREZ DE LA VEGA, M. 1988. Mating system in rye: Variability in relation to the population and plant density. Heredity 62, 17-26.

WRICKE, W. 1974. Seed set in rye after selfing under high temperature conditions. Incompt. Newsletter, 4, 23-26.

WRICKE, W. 1979. Degree of self-fertility under free pollination in rye populations containing a self-fertility gene. $Z$. Pflanzenzüchtg., 82, 281-285. 